\title{
Optical coherence tomography angiography study of the retinal vascular plexuses in type 1 diabetes without retinopathy
}

\author{
David Cordeiro Sousa $\mathbb{( D )}^{1,2,3} \cdot$ Inês Leal ${ }^{1,2}$. Susana Moreira ${ }^{4}$. Sónia do Vale $\mathbb{C}^{5}$ - Ana R. Silva-Herdade ${ }^{6}$. \\ Patrícia Dionísio ${ }^{4}$ Miguel A. R. B. Castanho $\mathbb{1}^{6} \cdot$ Luís Abegão Pinto $\mathbb{1}^{1,2} \cdot$ Carlos Marques-Neves ${ }^{1,2}$
}

Received: 26 February 2019 / Revised: 7 May 2019 / Accepted: 25 May 2019 / Published online: 4 July 2019

(c) The Author(s), under exclusive licence to The Royal College of Ophthalmologists 2019

\begin{abstract}
Aim Previous data suggest the existence of retinal vascular changes and impaired autoregulation in the very early stages of diabetic retinopathy (DR). We compared the retinal plexuses between patients with type 1 diabetes (T1D) without DR and a demographically similar healthy cohort, using optical coherence tomography angiography (OCT-A).

Methods Patients with T1D and no signs of DR were prospectively recruited from an outpatient clinic. Using OCT-A (AngioVue ${ }^{\circ}$ ), the parafoveal superficial (SCP) and deep (DPC) capillary plexus as well as the foveal avascular zone (FAZ) and perimeter were gathered. Mean comparison tests and linear regression analysis were used as statistical tests (STATA v14).

Results Studied population included 48 subjects (24 T1D). The analysis of SCP revealed an attenuation of the capillary network compared with the control group in both parafoveal $(51.8 \pm 4.5$ vs. $55.8 \pm 3.2, p<0.001)$ and perifoveal $(51.9 \pm 3.3$ vs. $53.9 \pm 1.9, p=0.01)$ regions. A similar finding was observed in the DCP for both parafoveal $(56.4 \pm 4.3$ vs. $60.4 \pm 2.2$, $p<0.001)$ and perifoveal $(54.7 \pm 3.9$ vs. $60.8 \pm 3.4, p=0.001)$ sectors. Also, a longer time since T1D diagnosis was associated with a larger FAZ area $(p=0.055)$ and perimeter $(p=0.03)$.

Conclusions Significant differences in the retinal microvasculature were observed between healthy subjects and T1D patients using OCT-A, even before clinically detectable disease on fundus biomicroscopy.
\end{abstract}

David Cordeiro Sousa

davidsousa@medicina.ulisboa.pt

1 Ophthalmology Department, Hospital de Santa Maria, Centro Hospitalar Universitário Lisboa Norte, Av. Professor Egas Moniz, 1649-035 Lisboa, Portugal

2 Vision Sciences Study Center, CECV, Faculdade de Medicina, Universidade de Lisboa, Av. Professor Egas Moniz, 1649-028 Lisboa, Portugal

3 Manchester Royal Eye Hospital, Manchester University Hospitals NHS Foundation Trust, Manchester, United Kingdom

4 Respiratory Medicine Department, Hospital de Santa Maria, Centro Hospitalar Universitário Lisboa Norte, Av. Professor Egas Moniz, 1649-035 Lisboa, Portugal

5 Endocrinology Department, Hospital de Santa Maria, Centro Hospitalar Universitário Lisboa Norte, Av. Professor Egas Moniz, 1649-035 Lisboa, Portugal

6 Instituto de Medicina Molecular, Faculdade de Medicina, Universidade de Lisboa, Av. Professor Egas Moniz, 1649-028 Lisboa, Portugal

\section{Introduction}

Diabetes mellitus and specifically diabetic retinopathy (DR) are major public health challenges [1]. Globally, roughly 400 million people have DM, and this number is expected to rise to more than 600 million by 2040 [2, 3]. Of these, type 1 diabetes (T1D) — which tends to be diagnosed in younger individuals-accounts for $\sim 5-10 \%$ of the cases [4]. As DR is a leading cause of acquired visual loss in the working-age population, an early and effective management of these patients is of paramount importance [5]. Previous data from studies in animal models [6] and humans [7] suggest the existence of retinal vascular changes and impaired autoregulation in the very early stages of DR [8].

Previous research with optical coherence tomography (OCT) provided evidence of inner retinal changes in patients with subclinical DR, suggesting that neurodegeneration accompanies early DR development $[9,10]$. Optical coherence tomography angiography (OCT-A) is a novel diagnostic tool that uses infrared wavelengths to provide non-invasive high-contrast high-resolution imaging of the 
retinal microvasculature [11-13]. It is able to detect motion contrast produced by moving blood cells in retinal vessels. Recent advances in projection artefact removal allowed to not only accurately define the superficial plexus but also the deep retinal vascular layers [14].

Although some studies report on OCT-A findings in type 2 diabetes, currently there is scarce data regarding early changes in younger patients with T1D [15-18]. Therefore, the aim of our study is to compare the superficial and deep retinal vessel plexuses between T1D patients without DR and a cohort of demographically similar healthy subjects using OCT-A technology.

\section{Methods}

This research protocol followed the tenets of the Declaration of Helsinki [19], and was submitted and approved by the Ethics Committee of Lisbon Academic Medical Center in March 2018. Written informed consent was obtained from all the participants before enrolment, after detailed explanation of the study.

\section{Participants}

A total of 27 adult subjects with T1D were prospectively recruited from an adult diabetes clinic. A demographically similar cohort of 24 healthy volunteers served as the control group. An anonymous questionnaire was filled out, including age, gender, smoking-pack years, diabetic disease duration and current treatment, other known medical history and current chronic medication. Subjects were asked to abstain from smoking, alcohol and caffeine for at least $6 \mathrm{~h}$ before the study to reduce potential autonomic effects and measurement bias [20].

Exclusion ophthalmological criteria for both groups were the presence of any degree of diabetic retinopathy on fundus examination, significant lens opacities, high refractive error (spherical equivalent below -6.50 or above +4.00 diopters), history of glaucoma or ocular hypertension and neuroophthalmic disease. Exclusion systemic criteria were hypertension, nephropathy or other documented microvascular complication, and smokers of more than five cigarettes a day. Pregnant women were also not recruited.

\section{Protocol}

A complete ophthalmological examination was conducted to all subjects, including best-corrected visual acuity (BCVA), slit-lamp biomicroscopy of the anterior and posterior segment, auto-refractometer and intraocular pressure (RK-5 ${ }^{\circ}$, Canon Europe ${ }^{\circ}$, The Netherlands), colour fundus photography (CR-2 ${ }^{\circ}$, Canon, USA) and optical biometry
(Lenstar ${ }^{\circledR}$, Haag-Streit, Switzerland). Glycated haemoglobin (HbA1c) values of T1D arm and time since diagnosis were retrieved from clinical notes.

OCT-A examination (AngioVue ${ }^{\bullet}$ Optovue, CA, USA) was performed by an experienced technician using the standard macular protocol. Parafoveal and perifoveal vessel densities in the superficial and deep plexuses were calculated automatically calculated using AngioAnalytics ${ }^{\circ}$, the built-in software of the OCT-A device. Foveal avascular zone area and perimeter were also calculated. Only high-quality images (score higher than 8/10) were considered. The device used was the latest version available, which includes the latest projection artefact removal algorithm [14].

\section{Sample size}

Based on previous OCT-A studies [21, 22], sample size was calculated considering a $10 \%$ clinically significant difference in vessel density between groups, and a standard deviation of $5 \%$. Accordingly, considering a power of $90 \%$, an alpha value of 0.05 , a minimum of 17 T1D patients and 17 controls were necessary to include.

\section{Statistical analysis}

Statistics were performed using STATA v14 (StataCorp LP, College Station, TX, USA). All quantitative data were expressed as mean \pm standard deviation. Comparisons of means between groups were done using Student's $t$ test. A multivariate linear regression model was used to assess correlation between continuous variables. $P$-values $<0.05$ were considered to be statistically significant.

\section{Results}

After excluding three T1D patients due to treated systemic hypertension, 48 subjects ( 24 T1D without DR and 24 healthy controls) were studied, with similar demographic and baseline ophthalmological characteristics (Table 1). In the T1D group, the time since diagnosis was $13.6 \pm 9.7$ [range 1-35] years, and mean $\mathrm{HbA1c}$ value was $8.0 \pm 1.4 \%$ [range 6.2-11.5\%]. Mean BCVA was 0.0 LogMar in both groups, and no significant abnormalities were found on ophthalmological examination of the included patients.

The analysis of superficial capillary plexuses revealed a rarefaction of the capillary network compared with the control group in both parafoveal $(51.8 \pm 4.5$ vs. $55.8 \pm 3.2$, $p<0.001)$ and perifoveal $(51.9 \pm 3.3$ vs. $53.9 \pm 1.9, p=$ $0.01)$ sectors. Similar findings were observed for deep capillary plexuses in both parafoveal $(56.4 \pm 4.3$ vs. $60.4 \pm 2.2, \quad p<0.001)$ and perifoveal $(54.7 \pm 3.9$ vs. $60.8 \pm 3.4, p=0.001$ ) sectors (Table 2). 
Table 1 Demographic and baseline data

\begin{tabular}{llll}
\hline & Control group & $\begin{array}{l}\text { Type 1 } \\
\text { diabetes }\end{array}$ & $p$-value \\
\hline Age, years & $31.8 \pm 8.2$ & $35.6 \pm 10.4$ & 0.17 \\
Male/female, $n$ & $10 / 14$ & $10 / 14$ & - \\
Body mass index, $\mathrm{kg} / \mathrm{m}^{2}$ & $22.6 \pm 3.0$ & $24.4 \pm 3.0$ & 0.12 \\
Intraocular & $13.3 \pm 2.1$ & $14.6 \pm 3.0$ & 0.14 \\
pressure, mmHg & & & \\
Axial length, mm & $24.1 \pm 0.9$ & $23.5 \pm 1.0$ & 0.07 \\
\hline
\end{tabular}

The data are expressed as mean \pm standard deviation, when applicable $P$-values obtained with Student's $t$ test

Table 2 OCT-angiography quantitative analysis of retinal vascular plexuses

\begin{tabular}{llll}
\hline & Control & $\begin{array}{l}\text { Type 1 diabetes } \\
\text { without DR }\end{array}$ & $p$-value \\
\hline Parafoveal & & & \\
$\begin{array}{l}\text { Superficial plexus } \\
\text { Deep plexus }\end{array}$ & $55.8 \pm 3.2$ & $51.8 \pm 4.5$ & $<0.001$ \\
Perifoveal & $60.4 \pm 2.2$ & $56.4 \pm 4.3$ & $<0.001$ \\
Superficial plexus & $53.9 \pm 1.9$ & $51.9 \pm 3.3$ & 0.01 \\
Deep plexus & $60.8 \pm 3.4$ & $54.7 \pm 3.9$ & 0.001 \\
FAZ area, mm ${ }^{2}$ & $0.23 \pm 0.10$ & $0.21 \pm 0.07$ & 0.52 \\
FAZ perimeter, mm & $1.83 \pm 0.44$ & $1.81 \pm 0.31$ & 0.83 \\
\hline
\end{tabular}

$D R$ diabetic retinopathy, $F A Z$ foveal avascular zone

The data are expressed as mean \pm standard deviation

$P$-values obtained with Student's $t$ test

No significant differences were found in mean FAZ area and perimeter when comparing diabetic and control groups. However, in an age-controlled linear regression model, a longer time since T1D diagnosis was associated with a larger FAZ area $(p=0.06)$ and perimeter $(p=0.03)$. Moreover, in T1D patients, a longer duration of disease was associated with decreased vessel densities in the parafoveal $(p=0.05)$ and perifoveal $(p=0.04)$ superficial plexuses, but not in the deep plexuses.

Age, gender and $\mathrm{HbAlc}$ values were not significantly correlated with any of the analysed outcomes.

\section{Discussion}

In this OCT-A study, a quantitative comparative analysis of different retinal vascular plexuses between patients with T1D without DR and a healthy cohort was performed. We were looking for the potential early vascular changes in T1D — even before clinically detectable disease — in order to better understand DR pathophysiology and ultimately improve the management of the disease.
Although OCT-A technology allowed us to noninvasively obtain images with unprecedented detail of the retinal microvasculature [11-13], some aspects still need improving. Specifically, one of its main recognised limitations has been the appearance of projection artefacts in deeper layers [14]. It should be mentioned that the device used in our study includes a projection artefact removal software, which allows to accurately define the deep retinal vascular layers, and not only the superficial plexus.

Our study reports the existence a significantly decreased macular vascular density in both superficial and deep plexuses in T1D patients without DR, comparatively with a healthy cohort. These early microvascular changes in T1D are in line with two studies published on the subject $[15,23]$. However, a recent study in a paediatric T1D population did not observe such difference [16]. Such variations are most probably justified by the significant differences in T1D duration among studies (13.6 \pm 9.7 years in our study vs. $11.0 \pm 4.0$ (15) vs. $6.4 \pm 6.2$ (16), being lowest in the latter study, in which no differences were found. Accordingly, we found that longer disease duration was associated with a decreased vessel density in superficial plexuses. Therefore, it is reasonable to think that the same paediatric population will eventually present similar OCT-A findings with a similar disease duration in the future.

As one of the most energy-demanding tissues in the body, the retina requires an effective blood flow regulation for its normal functioning [13]. It has the ability for local autoregulation, which is important to keep blood flow relatively constant, despite the variations in perfusion pressure [24]. This study suggests OCT-A is sensitive enough to detect significant microvascular changes in both superficial and deep retinal plexuses' before clinically detectable DR. These findings may correspond to an early stage of retinal blood flow autoregulation compromise, which may eventually lead to the sequence of events in the natural history of diabetic eye disease. As a complex disease, DR has been a field of controversy regarding relative contribution of each pathway and cascade into the overall outcome of vascular occlusion and retinal ischaemia [25]. The multitude of confounding factors such as advancing age, concomitant vascular conditions, accompanying macrovascular diseases, systemic medications that can interfere with autonomic nervous system response make this area of research a challenging one. Early diagnosed T1D are a subset of diabetic patients where these specific confounding aspects are minimised [26], thus allowing a more direct correlation between the retinal vascular response with the disease. Another strength of our results is the representative sample regarding the population demographics and wide range of time elapsed since T1D diagnosis.

Despite the mentioned differences observed in macular vascular density, FAZ area and perimeter were not different 
between the two groups. We hypothesise that a rarefaction in vessel density may precede the FAZ enlargement, which may be detectable only later in the course of the disease, as previously reported in type 2 diabetic patients with DR [27-29]. Another finding supporting this argument is the fact that we found a statistically significant association between longer T1D duration and larger FAZ area and perimeter. This suggests that changes in FAZ are also present before clinically detectable DR in patients with T1D. On the other hand, HbA1c values were not linearly associated with any of the outcomes. A clear explanation is lacking, but the fact that we used only one $\mathrm{HbAlc}$ value (and not mean values since diagnosis), the small standard deviation (i.e., a relatively homogeneous T1D sample), the fact that HbA1c does not measure glucose variability, and the multifactorial nature of the disease may all have contributed for the lack of significance.

Some study limitations should be acknowledged. Firstly, the study is cross-sectional in nature and although the series is larger than the calculated sample size, almost all patients were Portuguese and Caucasian (with the exception of two African T1D patients). Therefore, even being very likely to encounter similar findings in different populations and ethnicities, external validity is inherently limited. Secondly, OCT-A technology and the built-in analytics software calculate vessel density by image binarization, and its extrapolation as valid blood flow measurement is still to be fully validated, as in every OCT-A device. In the future, we suggest that exploring other vascular quantification methods and compare them with the reported OCT-A outcomes would provide new insights in this field.

\section{Conclusion}

Our work suggests there are significant differences in the retinal microvasculature between healthy subjects and T1D patients, even before clinically detectable disease on fundus biomicroscopy. OCT-A is a promising technology in the early diagnosis and management of diabetic eye disease.

\section{Summary}

\section{What was known before}

- Optical coherence tomography angiography (OCT-A) is a novel diagnostic tool, which allows non-invasive imaging of the retinal microvasculature with unpredecedent detail.

- In diabetic retinopathy (DR), OCT-A is being used to detect changes in retinal capillary plexuses, and an increasing rarefaction of retinal vessels has been described along with DR progression.

- It remains to be elucidated if early changes in retinal microvasculature could be detected before any visible clinical sign of DR on fundus biomicroscopy.

- Are retinal capillary plexuses different between type 1 diabetes patients without DR, and a cohort of demographically similar healthy subjects?

\section{What this study adds}

- Both deep and superficial retinal vessel densities were decreased in the very early stages of the disease, i.e., before any visible retinal changes on fundus examination.

- This work contributes to the current understanding of the pathophysiology of diabetic retinopathy, by revealing significant differences in the retinal microvasculature between healthy subjects and patients with type 1 diabetes, before the development of DR.

Acknowledgements The authors would like to thank José Cotta for providing the necessary medical equipment and logistic support for the study.

Funding The authors would like to thank AstraZeneca Foundation/ Faculty of Medicine of the University of Lisboa.

\section{Compliance with ethical standards}

Conflict of interest The authors declare that they have no conflict of interest.

Publisher's note: Springer Nature remains neutral with regard to jurisdictional claims in published maps and institutional affiliations.

\section{References}

1. Nentwich MM, Ulbig MW. Diabetic retinopathy -ocular complications of diabetes mellitus. World J Diabetes. 2015;6:489-99. http://www.wjgnet.com/esps/\%0Ahttp://www.wjgnet.com/esps/ helpdesk.aspx\%0Ahttp://www.wjgnet.\%0Ahttp://dx.doi

2. Ting DSW, Cheung GCM, Wong TY. Diabetic retinopathy: global prevalence, major risk factors, screening practices and public health challenges: a review. Clin Experiment Ophthalmol. 2015. http://doi.wiley.com/10.1111/ceo.12696

3. International Diabetes Federation. IDF diabetes atlas - Home. Eighth Edition. International Diabetes Federation, Brussels, Belgium. 2017. https://diabetesatlas.org/resources/how-to-cite.html.

4. You W-P, Henneberg M. Type 1 diabetes prevalence increasing globally and regionally: the role of natural selection and life expectancy at birth. BMJ Open Diabetes Res Care. 2016;4: e000161 http://www.ncbi.nlm.nih.gov/pubmed/26977306

5. Cheung N, Mitchell P, Wong TY. Diabetic retinopathy. Lancet. 2010;376:124-36. http://www.ncbi.nlm.nih.gov/pubmed/20580421 
6. Ivanova E, Kovacs-Oller T, Sagdullaev BT. Vascular pericyte impairment and connexin43 gap junction deficit contribute to vasomotor decline in diabetic retinopathy. J Neurosci. 2017;187-17. http://www.jneurosci.org/lookup/doi/10.1523/JNEUROSCI.018717.2017

7. Garhöfer G, Zawinka C, Resch H, Kothy P, Schmetterer L, Dorner GT. Reduced response of retinal vessel diameters to flicker stimulation in patients with diabetes. Br J Ophthalmol 2004;88:887-91.

8. Bianchi L, Porta C, Rinaldi A, Gazzaruso C, Fratino P, DeCata P, et al. Integrated cardiovascular/respiratory control in type 1 diabetes evidences functional imbalance: possible role of hypoxia. Int J Cardiol. 2017;1-6. https://doi.org/10.1016/j.ijcard.2017.06.047

9. Tavares Ferreira J, Proença R, Alves M, Dias-Santos A, Santos BO, Cunha JP, et al. Retina and choroid of diabetic patients without observed retinal vascular changes: a longitudinal study. Am J Ophthalmol. 2017;176:15-25.

10. El-Fayoumi D, Badr Eldine NM, Esmael AF, Ghalwash D, Soliman HM. Retinal nerve fiber layer and ganglion cell complex thicknesses are reduced in children with type 1 diabetes with no evidence of vascular retinopathy. Investig Opthalmology Vis Sci. 2016;57:5355-60.

11. Koustenis A, Harris A, Gross J, Januleviciene I, Shah A, Siesky B. Optical coherence tomography angiography: an overview of the technology and an assessment of applications for clinical research [Internet]. Br J Ophthal. 2017;101:16-20. http://www.ncbi.nlm. nih.gov/pubmed/27707691

12. Spaide RF, Fujimoto JG, Waheed NK, Sadda SR, Staurenghi G. Optical coherence tomography angiography. Prog Retin Eye Res. 2018;64:1-55. https://doi.org/10.1016/j.preteyeres.2017.11.003

13. Wei X, Balne PK, Meissner KE, Barathi VA, Schmetterer L, Agrawal R. Assessment of flow dynamics in retinal and choroidal microcirculation. Surv Ophthalmol. 2018;63:646-64.

14. Garrity ST, Iafe NA, Phasukkijwatana N, Chen X, Sarraf D. Quantitative analysis of three distinct retinal capillary plexuses in healthy eyes using optical coherence tomography angiography. Invest Ophthalmol Vis Sci. 2017;58:5548-55.

15. Carnevali A, Sacconi R, Corbelli E, Tomasso L, Querques L, Zerbini G, et al. Optical coherence tomography angiography analysis of retinal vascular plexuses and choriocapillaris in patients with type 1 diabetes without diabetic retinopathy. Acta Diabetol. 2017;54:695-702.

16. Gołębiewska J, Olechowski A, Wysocka-Mincewicz M, Odrobina D, Baszyńska-Wilk M, Groszek A, et al. Optical coherence tomography angiography vessel density in children with type 1 diabetes. PLoS One 2017;12:1-11.

17. Ishibazawa A, Nagaoka T, Takahashi A, Omae T, Tani T, Sogawa $\mathrm{K}$, et al. Optical coherence tomography angiography in diabetic retinopathy: a prospective pilot study. Am J Ophthalmol. 2015;160:35-44.e1.

18. Ting DSW, Tan GSW, Agrawal R, Yanagi Y, Sie NM, Wong $\mathrm{CW}$, et al. Optical coherence tomographic angiography in type 2 diabetes and diabetic retinopathy. JAMA Ophthalmol. 2017;135: 306-12.

19. Carlson RV, Boyd KM, Webb DJ. The revision of the Declaration of Helsinki: past, present and future. $\mathrm{Br} \mathrm{J}$ Clin Pharmacol. 2004;57:695-713.

20. Vinader-Caerols C, Monleón S, Carrasco C, Parra A. Effects of alcohol, coffee, and tobacco, alone or in combination, on physiological parameters and anxiety in a young population. J Caffeine Res. 2012;2:70-6

21. Xu H, Deng G, Jiang C, Kong X, Yu J, Sun X. Microcirculatory responses to hyperoxia in macular and peripapillary regions. Invest Ophthalmol Vis Sci. 2016;57:4464-8.

22. Kim AY, Chu Z, Shahidzadeh A, Wang RK, Puliafito CA, Kashani AH. Quantifying microvascular density and morphology in diabetic retinopathy using spectral-domain optical coherence tomography angiography. Invest Ophthalmol Vis Sci. 2016;57: 362-70.

23. Simonett JM, Scarinci F, Picconi F, Giorno P, De Geronimo D, Di Renzo A, et al. Early microvascular retinal changes in optical coherence tomography angiography in patients with type 1 diabetes mellitus. Acta Ophthalmol. 2017;95:e751-e755.

24. Arjamaa O, Nikinmaa M. Oxygen-dependent diseases in the retina: role of hypoxia-inducible factors. Experimental Eye Res. 2006;83:473-83.

25. Safi H, Safi S, Hafezi-Moghadam A, Ahmadieh H. Early detection of diabetic retinopathy. Surv Ophthalmol. 2018;63:601-8. https:// doi.org/10.1016/j.survophthal.2018.04.003

26. Song SH. Complication characteristics between young-onset type 2 versus type 1 diabetes in a UK population. BMJ Open Diabetes Res Care. 2015;3:e000044.

27. Scarinci F, Nesper PL, Fawzi AA. Deep retinal capillary nonperfusion is associated with photoreceptor disruption in diabetic macular ischemia. Am J Ophthalmol. 2016;168:129-38.

28. Freiberg FJ, Pfau M, Wons J, Wirth MA, Becker MD, Michels S. Optical coherence tomography angiography of the foveal avascular zone in diabetic retinopathy. Graefe's Arch Clin Exp Ophthalmol. 2016;254:1051-8.

29. Mastropasqua R, Toto L, Mastropasqua A, Aloia R, De Nicola $\mathrm{C}$, Mattei PA, et al. Foveal avascular zone area and parafoveal vessel density measurements in different stages of diabetic retinopathy by optical coherence tomography angiography. Int J Ophthalmol. 2017;10:1545-51. http://www.ncbi.nlm.nih.gov/ pmc/articles/PMC5638976/ 